\title{
INDEX FOR VOLUME 5, 2015
}

\author{
TABLE OF CONTENTS FOR VOLUME 5 \\ INTERNATIONAL JOURNAL FOR UNCERTAINTY QUANTIFICATION \\ Page Numbers for Issues:
}

Issue 1: 1-98; Issue 2: 99-193; Issue 3: 195-295;

Issue 4: 297-392; Issue 5: 393-489; Issue 6: 491-583

\section{ISSUE 1}

Minimal Sparse Sampling for Fourier-Polynomial Chaos in Acoustic Scattering Roger M. Oba

A Mixed Uncertainty Quantification Approach using Evidence Theory and Stochastic Expansions 21 Harsheel Shah, Serhat Hosder, \& Tyler Winter

A Gradient-Based Sampling Approach for Dimension Reduction of Partial Differential Equations with Stochastic Coefficients Miroslav Stoyanov \& Clayton G. Webster

Bayesian Inference for Inverse Problems Occurring in Uncertainty Analysis

Shuai Fu, Gilles Celeux, Nicolas Bousquet, Mathieu Couplet

\section{ISSUE 2}

A Nonstationary Covariance Function Model for Spatial Uncertainties in Electrostatically Actuated Microsystems Aravind Alwan, Narayana R. Aluru

Bivariate Quantile Interpolation for Ensemble Derived Probability Density Estimates Brad Eric Hollister, Alex Pang

Hierarchical Sparse Bayesian Learning for Strucutral Health Monitoring with Incomplete Modal Data Yong Huang, James L. Beck

Polynomial-Chaos-Based Kriging Roland Schobi, Bruno Sudret, Joe Wiart

\section{ISSUE 3}

Preface

A. Chernov \& F. Nobile

Uncertainty Quantification for Maxwell's Equations Using Stochastic Collocation and Model Order Reduction P. Benner \& J. Schneider

Second-Order Sensitivity Analysis of Parameter Estimation Problems E. Kostina \& M. Nattermann

A Hermite Spectral Method for a Fokker-Planck Optimal Control Problem in an Unbounded Domain M. Mohammadi \& A. Borzi

Stochastic Galerkin Methods and Model Order Reduction for Linear Dynamical Systems R. Pulch \& E. J. W. ter Maten

An Optimal Sampling Rule for Nonintrusive Polynomial Chaos Expansions of Expensive Models 275 M. Sinsbeck \& W. Nowak 


\section{ISSUE 4}

Probabilistic Quantification of Hazards: A Methodology using Small Ensembles of PhysicsBased Simulations and Statistical Surrogates

M.J. Bayarri, J.O. Berger, E.S. Calder, A K. Patra, E.B. Pitman, E.T. Spiller, \& R.L. Wolpert

Uncertainty Quantification of the GEM Challenge Magnetic Reconnection Problem using the Multilevel Monte Carlo Method

É.M. Sousa, G. Lin, \& U. Shumlak

Surrogate Preposterior Analyses for Predicting and Enhancing Identifiability in Model Calibration Z. Jiang, D.W. Apley, \& W. Chen

A New Gibbs Sampling Based Bayesian Model Updating Approach using Modal Data from Multiple Setups S. Bansal

Low-Cost Multi-Dimensional Gaussian Process with Application to Uncertainty Quantification

\section{ISSUE 5}

High Dimensional Sensitivity Analysis using Surrogate Modeling and High Dimensional Model Representation M. Kubicek, E. Minisci, \& M Cisternino

A Posteriori Error Estimation for a Cut Cell Finite Volume Method with Uncertain Interface Location J.B. Collins, D. Estep, \& S. Tavener

An Uncertainty Visualization Technique using Possibility Theory: Possibilistic Marching

Cubes Y. He, M. Mirzargar, S. Hudson, R.M. Kirby, \& R.T. Whitaker

A Gradient-Enhanced Sparse Grid Algorithm for Uncertainty Quantification J.H.S. de Baar \& B. Harding

Random Predictor Models for Rigorous Uncertainty Quantification L.G. Crespo, S.P. Kenny, \& D.P. Giesy

\section{ISSUE 6}

An Error Subspace Perspective on Data Assimilation

Bayesian Approach to the Statistical Inverse Problem of Scatterometry: Comparison of Three Surrogate Models S. Heidenreich, H. Gross, \& M. Bär

A Block Circulant Embedding Method for Simulation of Stationary Gaussian Random Fields on Block-Regular Grids M. Park \& M. V. Tretyakov

Optimal Sensor Placement for the Estimation of Turbulence Model Parameters in CFD 545

D. I. Papadimitriou \& C. Papadimitriou

Robustness of Wilks' Conservative Estimate of Confidence Intervals 


\section{Author Index to Volume 5}

\section{INTERNATIONAL JOURNAL FOR UNCERTAINTY QUANTIFICATION \\ Page Numbers for Issues:}

Issue 1: 1-98; Issue 2: 99-193; Issue 3: 195-295;

Issue 4: 297-392; Issue 5: 393-489; Issue 6: 491-583

\author{
Aluru, N.R., 99 \\ Alwan, A., 99 \\ Apley, D.W., 341 \\ Bansal, S., 361 \\ Bär, M., 511 \\ Bayarri, M.J., 297 \\ Beck, J.L., 139 \\ Benner, P., 195 \\ Berger, J.O., 297 \\ Borzi, A., 233 \\ Bousquet, N., 73 \\ Calder, E.S., 297 \\ Celeux, G., 73 \\ Chen, W., 341 \\ Cheng, H., 491 \\ Chernov, A., vii \\ Cisternino, M., 393 \\ Collins, J.B., 415 \\ Couplet, M., 73 \\ Crespo, L.G., 469 \\ de Baar, J.H.S., 453 \\ Estep, D., 415 \\ $\mathrm{Fu}, \mathrm{S}$., 73 \\ Giesy, D.P., 469 \\ Gross, H., 511
}

Harding, B., 453

He, Y., 433

Heidenreich, S., 511

Hessling, J.P., 569

Hollister, B.E., 123

Hosder, S., 21

Huang, Y., 139

Hudson, S., 433

Jiang, Z., 341

Kenny, S.P., 469

Kirby, R.M., 433

Konomi, B.A., 375

Kostina, E., 209

Kubicek, M., 393

Lin, G., 327, 375

Minisci, E., 393

Mirzargar, M., 433

Mohammadi, M., 233

Nattermann, M., 209

Nobile, F., vii

Nowak, W., 275

Oba, R.M., 1

Pang, A., 123

Papadimitriou, C., 545
Papadimitriou, D.I., 545

Park, M., 527

Patra, A.K., 297

Pitman, E.B., 297

Pulch, R., 255

Sandu, A., 491

Schneider, J., 195

Schobi, R., 171

Shah, H., 21

Shumlak, U., 327

Sinsbeck, M., 275

Sousa, E.M., 327

Spiller, E.T., 297

Stoyanov, M., 49

Sudret, B., 171

Tavener, S., 415

ter Maten, E. Jan W., 255

Tretyakov, M.V., 527

Uhlmann, J., 569

Webster, C.G., 49

Whitaker, R.T., 433

Wiart, J., 171

Winter, T., 21

Wolpert, R.L., 297 


\section{SUBJECT INDEX TO VOLUME 5}

\section{INTERNATIONAL JOURNAL FOR UNCERTAINTY QUANTIFICATION}

\section{Page Numbers for Issues:}

Issue 1: 1-98; Issue 2: 99-193; Issue 3: 195-295;

Issue 4: 297-392; Issue 5: 393-489; Issue 6: 491-583

acoustics, 1

arbitrary distribution, 275

assessment error, 73

Bayesian analysis, 73

Bayesian inference, 99, 341, 511, 545

Bayesian model updating, 361

Bayesian Ockham razor, 139

benchmark functions, 171

block fast-fourier transform, 527

circulant embed-collocation, 527

computational fluid dynamics, 21

computational statistics, 123

computer codes (experiments), 375

computer modeling, 297

cut-HDMR, 393

damage detection, 139

data assimilation, 491

density estimation, 123

dependent parameters, 275

design of experiments, 73

ding method, 527

dynamical system, 139, 255

electromagnetics, 195

ensemble filters, 491

evaluation, 569

evidence theory, 21

Fokker-Planck equation, 233

Gaussian process modeling, 171

Gibbs sampling, 361

hazard assessment, 297

Hermite spectral discretization, 233

hierarchical sparse Bayesian learning, 139

high-dimensional approximation, 49

high-dimensional methods, 1

high-dimensional space, 393

high-dimensional surrogates, 453

hybrid methods, 491

IASC-ASCE simulated Benchmark problem, 139

information entropy, 545

inverse problem, 73, 139, 209, 511

irregular grids, 527

isosurface extraction, 433

Karhunen-Loeve expansion, 49

Kriging, 73, 171 linear model of coregionalization, 375

magnetic reconnection, 327

marching cubes, 433

Markov chain Monte Carlo, 375

material uncertainties, 195

maximum likelihood, 209

meta-modeling, 171

microelectromechanical systems (MEMS), 99

model calibration, 341

model order reduction, 195, 255

modeling, 569

Monte Carlo, 49, 327

multi-level Monte Carlo, 327

multiple setups, 361

multivariate Gaussian process, 375

nested quadrature rules, 275

nonstationary covariance function, 99

optimal control theory, 233

optimization, 469

parameter estimation, 209, 341, 469

PC-Kriging, 171

point-collocation, 21

polynomial chaos, 1, 21, 255, 275

polynomial chaos expansions, 171

porous media flow, 415

possibilistic marching cubes, 433

possibility theory, 433

probabilistic collocation, 327

propagation, 569

quadrature, 255

quasi-neutral ion cyclotron wave, 327

random fields, 123

random process, 99, 469

representation of uncertainty, 49, 21, 123, 433, 469

representing of uncertainty, 209

robustness, 545

sampling, 569

sampling techniques, 527

scatterometry, 511

sensitivity analysis, 393

separability, 375

Smolyak algorithm, 1

sparse grid, 195, 453

spatial statistics, 123 
spatial uncertainty, 99, 123, 415

stationary Gaussian random field, 527

statistics, 297

stochastic collocation, 195, 275

stochastic Galerkin method, 255

stochastic model reduction method, 49

stochastic modeling, 255, 415

stochastic partial differential equations, 1, 49, 511

stochastic response surface method, 469

stochastic sensitivity analysis, 49, 209

stochastic simulation, 361 stochastic sparse grid collocation, 1

structural health monitoring, 139

symmetric block-toeplitz matrices, 527

turbulence models, 545

two-fluid plasma model, 327

uncertainty, 297, 569

uncertainty quantification, 21, 99, 123, 195, 209,

255, 275, 341, 361, 393, 415, 453, 469

uncertainty visualization, 433

variational methods, 491

volcanic hazards, 297 